\title{
Notch1 destabilizes the adherens junction complex through upregulation of the Snail family of E-cadherin repressors in non-small cell lung cancer
}

\author{
ARUM KIM ${ }^{1,2^{*}}$, EUN YOUNG KIM ${ }^{1 *}$, EUN NA CHO ${ }^{1}$, HYUNG JUNG KIM $^{1}$, SE KYU KIM ${ }^{1}$, \\ JOON CHANG ${ }^{1}, \mathrm{CHUL} \mathrm{MIN} \mathrm{AHN}^{1}$ and YOON SOO CHANG ${ }^{1,2}$ \\ ${ }^{1}$ Department of Internal Medicine and ${ }^{2}$ Biomedical Research Institute, \\ Yonsei University College of Medicine, Seoul, Republic of Korea
}

Received March 14, 2013; Accepted May 29, 2013

DOI: $10.3892 /$ or.2013.2565

\begin{abstract}
One of the critical steps driving cancer cell migration and metastasis is the repression of cell adhesion molecules resulting in loss of cell-to-cell adhesion. Although interactions between Notch1 and components of the adherens junction complex have been suggested, little is known concerning the consequence of their interactions. In this study, we investigated the interaction between the Notch 1 and the E-cadherin/ $\beta$-catenin complex, its effect on the expression of adherens junction complex components and its influence on non-small cell lung cancer (NSCLC) cell proliferation. With progression of lung neoplastic lesions in LSL K-ras G12D mice, the expression of E-cadherin was inhibited whereas that of Notch1 was increased with frequent nuclear localization, suggesting an inverse relationship between E-cadherin and Notch1 expression with tumor progression. Transduction of the human Notch1 intracellular domain (N1ICD) into NSCLC cells inhibited expression of E-cadherin and $\beta$-catenin and induced changes in the localization of adherens junction molecules. The loss of E-cadherin was mediated through upregulation of the Snail family of transcription factors, Snail and Slug. Experiments in which siRNA against E-cadherin was introduced into NSCLC cells revealed that N1ICD decreased the expression of $\beta$-catenin in an E-cadherin-independent manner, leading to inhibition of markers of $\mathrm{Wnt} / \beta$-catenin signaling activation. Despite inhibition of Wnt/ $\beta$-catenin signaling in the N1ICD-transduced cells, cells transduced with N1ICD showed no difference in cell cycle progression when compared with that of the control vector-
\end{abstract}

Correspondence to: Dr Yoon Soo Chang, Department of Internal Medicine, Yonsei University College of Medicine, 8th Floor, Annex Building, 211 Eonju-ro, Gangnam-gu, Seoul 135-720, Republic of Korea

E-mail: yschang@yuhs.ac

*Contributed equally

Key words: Notch1, adherens junction complex, E-cadherin, E-cadherin repressor complex, Wnt $/ \beta$-catenin, non-small cell lung cancer transduced cells. In conclusion, Notch1 inhibited the expression of E-cadherin through upregulation of the Snail family of transcriptional factors, resulting in inhibition of expression of $\beta$-catenin and destabilization of adherens junctions.

\section{Introduction}

According to the 2011 annual report on the global burden of cancer, 1,600,000 new cases of lung cancer were noted in 2008 , which accounted for $13 \%$ of the total cancer cases worldwide, and there were 1,400,000 lung cancer-related deaths during the same period, which accounted for $18 \%$ of all cancer-related mortality making lung cancer the leading cause of cancer-related death worldwide (1). Lung cancer has a very poor prognosis compared with other cancer types, indicating that there is an urgent need for new therapies for non-small cell lung cancer (NSCLC) by elucidating the mechanisms of its growth and progression.

The single-pass heterodimeric transmembrane receptor protein, Notch1, localizes on the cell surface and mediates cell-to-cell interactions. When the extracellular domain of Notch1 dissociates from the heterodimerization domain through interaction with ligand cells, the Notch1 receptor undergoes sequential proteolytic cleavage and the Notch1 intracellular domain (N1ICD) is released and translocates to the nucleus where it activates transcription of target genes (2). During the neoplastic process, the tumor microenvironment becomes more solid and hypoxic resulting in intensified interactions between cells, which eventually generate conditions with amplified Notch1 signaling $(3,4)$.

Although the oncogenic role of Notch1 has been established in T-acute lymphoblastic leukemia, its roles in other neoplastic diseases are controversial (5). In T-cells, N1ICD induces the expression of cyclin D3, CDK4 and CDK6, leading to G1-S cell cycle phase progression (6). Other authors have reported that Notch1 signaling inhibits B lymphocyte growth (7), small cell lung cancer (8) and hepatocellular carcinoma (9). Since deregulated Notch1 signaling is common in NSCLC $(10,11)$ and several $\gamma$-secretase inhibitors (GSIs) are under development for various clinical applications, clarification of its role is required. 
Among the intercellular junctional complexes which maintain epithelial integrity and polarity, adherens junctions are made up of homophillic interactions between E-cadherin molecules and $\alpha$-catenin, $\beta$-catenin and $\delta 1$-catenin (p-120), which are connected to the actin cytoskeletal network. Interaction between E-cadherin and $\beta$-catenin stabilizes cell-to-cell contacts and maintains adherens junctions, and is important for cell-to-cell interaction-mediated signaling (12). Destabilization of adherens junctions facilitates endocytosis of E-cadherin and changes in the levels of $\beta$-catenin in various cellular compartments. Disruption of E-cadherin/ $\beta$-catenin complexes also decreases intercellular adhesion and further increases cell migration and invasiveness $(13,14)$. Physical interaction of Notch 1 with $\beta$-catenin through the RAM domain of Notch1 suggests another role of Notch1 in maintaining the stability of adherens junctions (15). By elucidating the function of Notch1 in adherens junctions, the context-dependent role of Notch1 may become clear.

In the present study, we investigated the role of Notch1 in adherens junction stability and NSCLC cell growth. Overexpression of N1ICD decreased E-cadherin through upregulation of the snail family of transcription repressors and it resulted in the alteration of total and active $\beta$-catenin expression. We found that Notch1 and $\beta$-catenin interact independently to E-cadherin expression and that Notch1 deregulates Wnt/ $\beta$-catenin signaling. Our results suggest that Notch1 is an important component involved in the physical and signaling homeostasis of adherens junctions.

\section{Materials and methods}

Plasmids, antibodies and cell cycle analysis. A549 cells were purchased from ATCC (Manassas, VA, USA). H460, H596 and H1650 cells were obtained from the Korean Cell Line Bank (Seoul, Korea). pMSCV-myc-N1ICD and -control vectors were obtained from Dr G. Jung (Seoul National University) and transduced as previously described (5). pCS2-ICV-6mt and pCS2- $\Delta$ EMV-6mt were gifts from Dr R. Kopan (http://devbio.wustl.edu/kopannulab/plasmids. htm) (16). Anti-Notch1 (C-20)-R and (C-10), -HES1, -HERP, -c-Myc were purchased from Santa Cruz Biotechnology, Inc. (Santa Cruz, CA, USA), and antibodies, unless otherwise stated, were obtained from Cell Signaling Technology (Danvers, MA, USA). For cell cycle analysis, cells were serum starved for $36 \mathrm{~h}$ and then released from cell cycle arrest by replacing the RPMI media supplemented with 10\% FBS. Cells were stained with propidium iodide and analyzed using a FACSCanto II flow cytometer (Becton-Dickinson, Franklin Lakes, NJ, USA).

Animal model and immunohistochemistry (IHC). Lox-Stop-Lox (LSL) K-ras G12D mice were obtained from the NCI mouse repository (http://mouse.ncifcrf.gov/), bred, and genotyped according to the supplier's guidelines. This animal study was approved by our Institutional Animal Care and Use Committee, following the guidelines of the American Association for the Assessment and Accreditation of Laboratory Animal Care. AdCre virus was obtained from the Gene Transfer Vector Core of the University of Iowa (Iowa City, IA, USA). Eight-week-old heterozygotes were intranasally infected with $5 \times 10^{8} \mathrm{PFU}$ of the AdCre virus according to a protocol described elsewhere (17). Six, 12 and 16 weeks after infection, mice were sacrificed, and expression of E-cadherin and Notch1 were analyzed using IHC. IHC was performed using the LABS ${ }^{\circledR} 2$ System (Dako, Carpinteria, CA, USA) according to the manufacturer's instructions. Briefly, sections were deparaffinization, rehydrated, immersed in $\mathrm{H}_{2} \mathrm{O}_{2}$-methanol solution, and then incubated overnight with primary anti-E-cadherin or -Notch1 antibodies in antibody diluent (Dako) at a 1:100 dilution. Sections were incubated for 10 min with biotinylated linker and processed using avidin-biotin IHC techniques. 3,3'-Diaminobenzidine (DAB) was used as a chromogen in conjunction with the Liquid DAB Substrate kit (Novacastra, UK).

Quantitative real time ( $R T)$ - $P C R$. Total RNA was extracted using TRI reagent ${ }^{\circledR}$ (Ambion, Austin, TX, USA). Quantitative RT-PCR analysis was conducted using TaqMan ${ }^{\circledR}$ Gene Expression assay reagents and the StepOnePlus ${ }^{\mathrm{TM}}$ Real-Time PCR system (Applied Biosystems, Carlsbad, CA, USA) using an inventoried primer-probe set described in the company's websites (http://bioinfo.appliedbiosystems.com/genome-database/gene-expression.html).

EDTA treatment and siRNA experiment. Induction of N1ICD and activation of Notch signaling using calcium treatment was described elsewhere $(10,18)$. Briefly, cells were washed two times with $\mathrm{Ca}^{2+}$ - and $\mathrm{Mg}^{2+}$-free DPBS and then incubated with DPBS containing $2.5 \mathrm{mM}$ EDTA for $5 \mathrm{~min}$. The media were then replaced with full growth media, and cells were incubated for the indicated times. MISSON ${ }^{\circledR}$ Predesigned siRNA against E-cadherin was obtained from Sigma-Aldrich (St. Louis, MO, USA) and transfected using Lipofectamine ${ }^{\circledR}$ RNAiMAX (Invitrogen, Carlsbad, CA, USA) reagents as per the manufacturer's recommendations.

Western blotting. Cells were harvested using 2X LSB lysis buffer containing protease inhibitor cocktail and phosphatase inhibitor cocktail (Sigma-Aldrich) on ice. After sonication, the BCA protein assay reagent (Thermo Scientific, Rockford, IL, USA) was used for protein quantification. Protein lysates (30-50 mg) were separated by gel electrophoresis on 7.5 to $12 \%$ polyacrylamide gels and analyzed by western blot analysis using nitrocellulose membranes (Bio-Rad Laboratories, Inc., Richmond, CA, USA). The expression level of each protein was measured using ImageJ (http://rsbweb.nih.gov/ij/) and quantified relative to that of $\beta$-actin.

Immunocytochemistry. Cells $\left(5 \times 10^{5}\right)$ were plated in 6-well plates containing a sterilized coverslip. On the following day, cells were fixed with $4 \%$ formaldehyde in PBS, incubated in blocking solution containing 5\% BSA in PBS, and then anti-c-Myc mouse (1:100) and $\alpha$-E-catenin, $\beta$-catenin, $\delta 1$-catenin rabbit antibodies (1:200) were added for $16 \mathrm{~h}$. On the following day, the cells were washed, and anti-mouse IgG (Alexa Fluor 555 conjugate) and anti-rabbit IgG (Alexa Flour 488 conjugate) secondary antibodies were added. The nuclei were counterstained with DAPI $(1: 1,000)$ in PBS and imaged using an LMS 510 confocal microscope (Carl Zeiss, Oberkochen, Germany). 

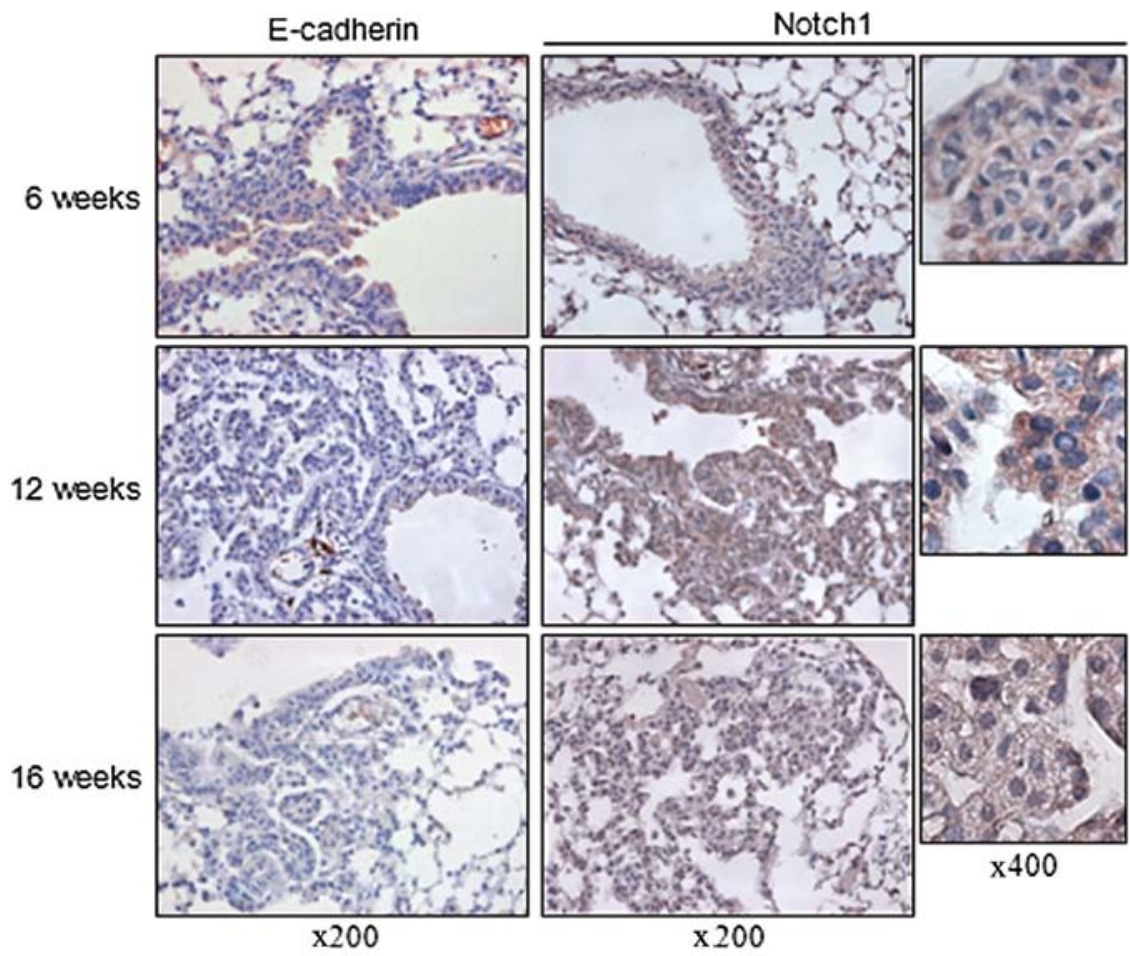

Figure 1. Inverse relationship between E-cadherin and Notch1 expression in a lung cancer mouse model. Eight-week-old LSL-K-ras G12D mice, which carry a latent point-mutant allele of Kras2 (K-ras G12D) under the control of a transcriptional termination sequence (Lox-Stop-Lox), underwent inhalation of $5 \times 10^{8}$ PFU AdCre virus intranasally and were then sacrificed at the indicated times. Expression of E-cadherin and Notch1 was evaluated by immunohistochemistry. Expression of E-cadherin was decreased over time whereas that of Notch1 was increased in both the cytoplasm and nucleus of the neoplastic lesions.

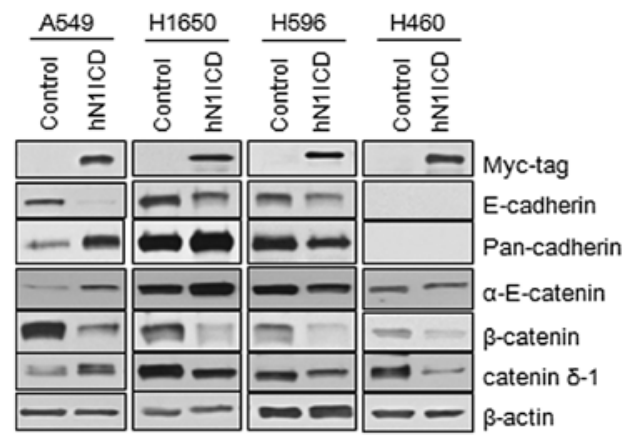

Figure 2. Transduction of Notch1 intracellular domain (N1ICD) decreases the expression of E-cadherin and $\beta$-catenin. Various non-small cell lung cancer cell lines, which express E-cadherin (A549, H1650 and H596) and which do not express E-cadherin (H460), were transduced with N1ICD using an MSCV retroviral vector and the expression of molecules comprising the adherens junction complex was evaluated by immunoblotting. A decrease in E-cadherin and $\beta$-catenin expression by N1ICD transduction was observed in A549, H1650 and H596 cells.

Statistical analysis. The independent sample t-test was used for univariate analysis of continuous variables. All statistical analyses were two-tailed.

\section{Results}

Inverse relationship between E-cadherin and Notch1 expression in a lung cancer mouse model. E-cadherin is an active suppressor of tumor growth and loss of its expression is related to the invasion, metastasis and poor prognosis of cancer whereas the activity of Notch1 signaling correlates with increased intercellular interaction and a hypoxic condition which are related to tumor progression (4,19-21). Therefore, we postulated that there is an inverse relationship between E-cadherin and Notch1 expression. To investigate this, 8-weekold LSL K-ras G12D mice were administered the AdCre virus intranasally and sacrificed at 6,12 and 16 weeks after viral inhalation. E-cadherin expression decreased with the progression of tumors over time. Compared to the hyperplastic lesions of the mice sacrificed at 6 weeks after AdCre virus inhalation, lesions of the mice sacrificed at 12 and 16 weeks after inhalation showed loss of E-cadherin expression. Notch1 was ubiquitously expressed in the membranous lesions of the normal-appearing bronchial epithelial cells and hyperplastic lesions of the lungs of mice sacrificed at 6 weeks after AdCre virus inhalation. In the neoplastic lesions from the mice which were sacrificed at 12 weeks after virus inhalation, there was increased cytoplasmic expression of Notch1. More frequent nuclear expression of Notch1, which is considered as biologically active, was detected in the neoplastic lesions of the lungs from the mice sacrificed at 16 weeks after inhalation. These findings suggest an inverse relationship between E-cadherin and Notch1 expression with the progression of tumors of LSL K-ras G12D mice (Fig. 1).

Transduction of N1ICD induces changes in the components of adherens junctions. To evaluate the role of Notch1 signaling on adherens junctions, we first transduced either the human N1ICD-myc or the control vector into NSCLC cells and assessed the expression of components of adherens junctions by immunoblotting. The characteristics of the MSCV-N1ICD-myc and MSCV-control vectors are described 


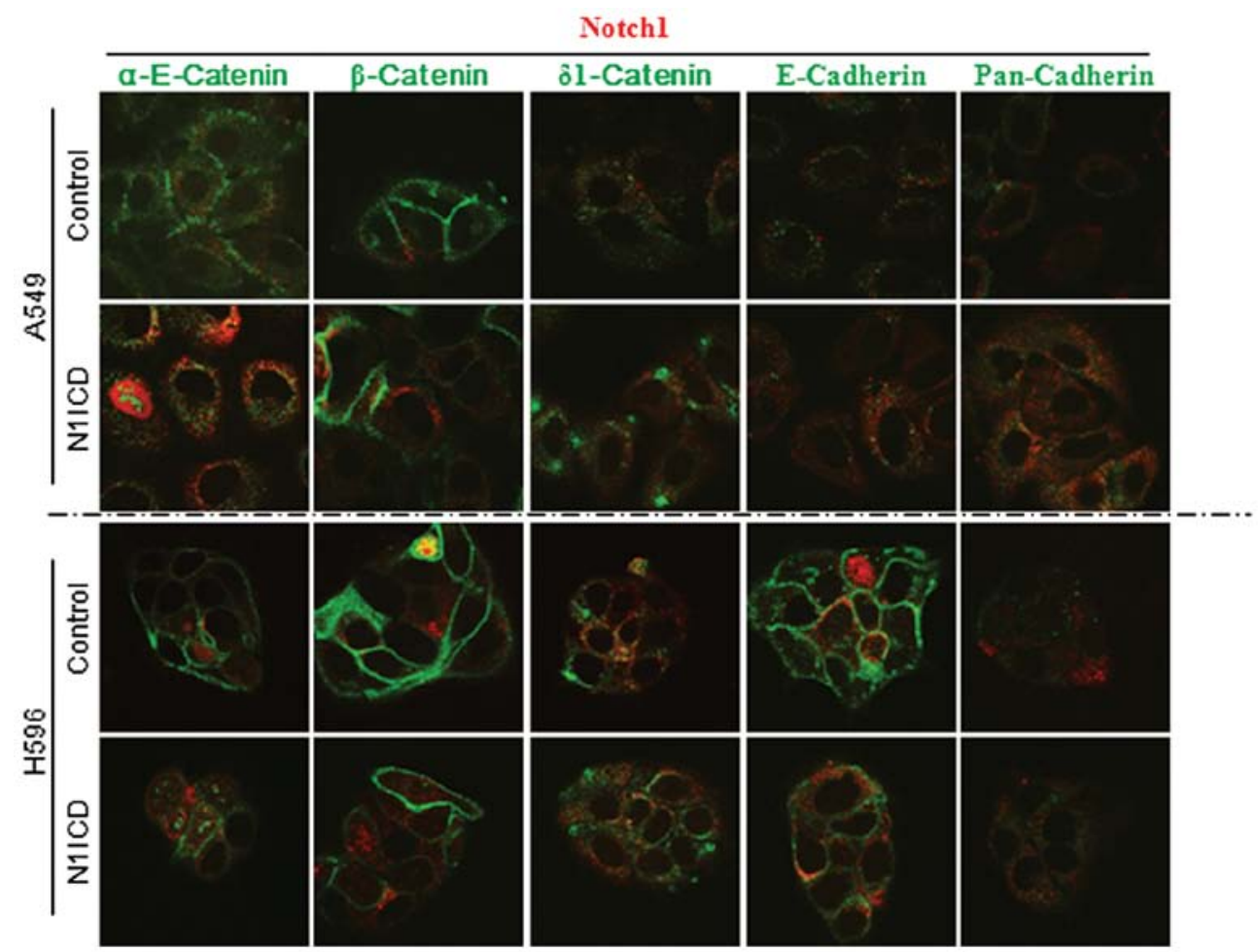

Figure 3. Notch1 intracellular domain (N1ICD) transduction alters the distribution of adherens junction components of non-small cell lung cancer (NSCLC) cells. Control and N1ICD vector-transduced NSCLC cells were stained for Notch1 (Red color) and adherens junction components, $\alpha$-E catenin, $\beta$-catenin, $\delta 1$-catenin, E-cadherin and pan-cadherin (green color), and then imaged using confocal microscopy $(\mathrm{x} 1,000)$. Transduction of N1ICD induced changes in both signal intensities and distribution of adherens junction components when compared with those transduced by the control vector.

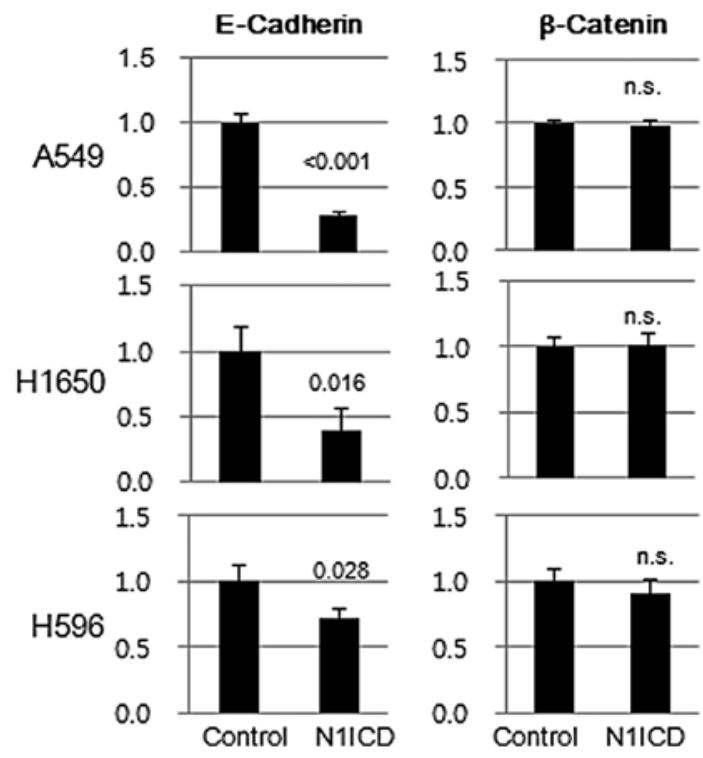

Figure 4. Change in E-cadherin is attributed to transcriptional repression but that of $\beta$-catenin is attributed to the post-translational level. mRNA levels of E-cadherin and $\beta$-catenin in either the control or Notch1 intracellular domain (N1ICD) vector-transduced non-small cell lung cancer (NSCLC) cells were evaluated by real-time PCR. NSCLC cells transduced with N1ICD showed a decreased level of E-cadherin mRNA compared with that of the control vector, but there was no difference in the mRNA levels of $\beta$-catenin Comparisons were made to that of cells transduced with the control vector. P-value was obtained by Student's t-test. n.s., not significant.

in Lim et al (5). Transduction of N1ICD was confirmed by expression of the myc tag at the $\mathrm{N}$-terminal of the insert and the overexpression of HES1 and Herp (data not shown). A549, H1650 and H596 cells, which express E-cadherin, and H460 cells, which do not express E-cadherin, showed inhibition of E-cadherin and $\beta$-catenin expression. Other components of the adherens junction, $\alpha$-E-catenin and $\delta 1$-catenin, showed different changes depending on the cell lines (Fig. 2). To further evaluate the effect of Notch signaling on adherens junctions, we performed an immunocytochemical study using N1ICD- and control vector-transduced cells. Transduction of N1ICD showed decreased membranous localization of $\alpha$-E-catenin, $\beta$-catenin and E-cadherin in A549, H596 (Fig. 3) and H1650 NSCLC cells (data not shown). Transduction of N1ICD inhibited both protein and mRNA expression of E-cadherin in A5459, H1650 and H596 cells. However, there was no difference in $\beta$-catenin mRNA expression between N1ICD- and control vector-transduced cells, which suggests post-translational regulation of $\beta$-catenin expression (Fig. 4).

Notch1 inhibits E-cadherin expression through Snail family of transcriptional complex. Calcium chelating agents interfere with the non-covalent binding of the Notch1 ectodermal domain to the Notch1 transmembrane intracellular (NTMIC) domain and facilitate S2 and S3 cleavage of Notch1 to activate Notch signaling (10). Brief exposure to the millimolar concentrations of EDTA resulted in the robust induction of N1ICD in a short period of time, and the signal was propagated to Notch target molecules, namely HES1 and Herp. Pretreatment of cells with compound E, a $\gamma$-secretase inhibitor, inhibited the induction of N1ICD and its target molecules (Fig. 5A). HES1 expression was upregulated at the transcriptional level 
A

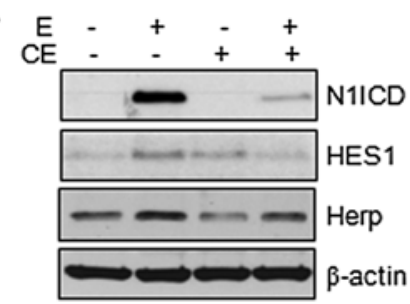

C
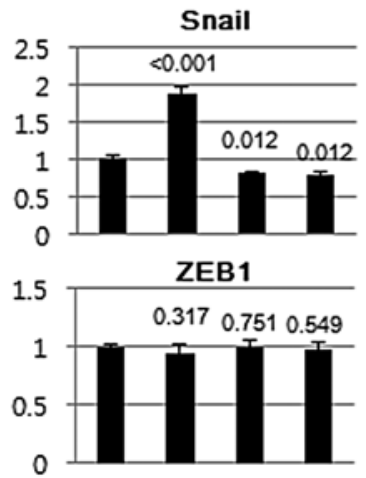

TWIST1

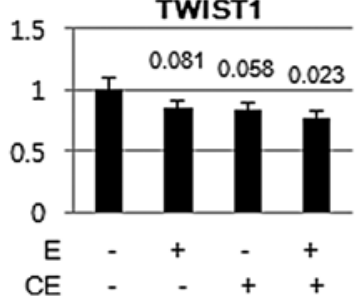

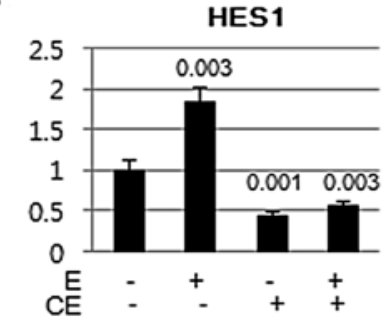

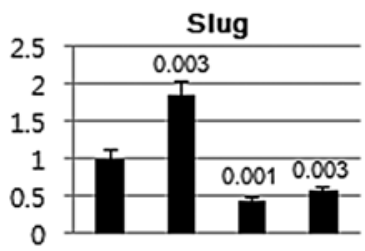

ZEB2

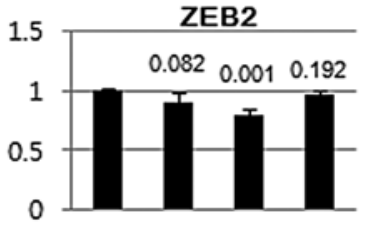

TCF3

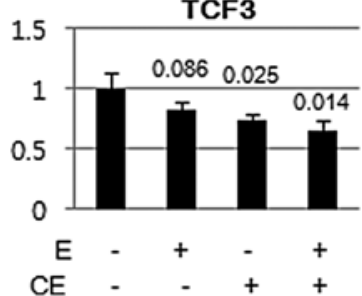

Figure 5. Snail family of E-cadherin repressor complex, Snail and Slug, are induced by Notch1 intracellular domain (N1ICD). (A) A549 cells were exposed to EDTA $(2.5 \mathrm{mM})$ for $5 \mathrm{~min}$ and expression of N1ICD and its downstream readouts of Notch1 signaling, HES1 and Herp, were evaluated by immunoblotting. Brief exposure to the calcium chelating agent, EDTA, induced the expression of N1ICD and its downstream targets, HES1 and Herp, whereas pretreatment of a $\gamma$-secretase inhibitor, compound $E$, inhibited the expression of N1ICD and its downstream targets. (B) Real-time PCR showed that induction of HES1 by EDTA and inhibition by compound E were regulated at the transcriptional level. (C) Real-time PCR of members of the E-cadherin repression complexes. Transcript expression of Snail and Slug was consistently increased by EDTA treatment and was inhibited by compound E. Comparisons were made to that of cells treated with vehicle. P-value was obtained by Student's t-test. CE, compound E; E, EDTA.

(Fig. 5B). Since induction of E-cadherin repressor complexes is one of the important mechanisms that induces E-cadherin loss, we evaluated the mRNA expression of molecules that comprise the E-cadherin repressor complex by RT-PCR. Among the E-cadherin repressor complex molecules, both Snail and Slug were the E-cadherin repressor complex that showed consistent increase in response to N1ICD induction (Fig. 5C).

N1ICD-induced inhibition of $\beta$-catenin is E-cadherin independent. Since the transduction of N1ICD into H460 cells, which do not express E-cadherin, showed a decrease in $\beta$-catenin expression, we aimed to ascertain whether the Notch1-mediated inhibition of $\beta$-catenin expression was E-cadherin dependent. We treated A549 cells with siRNA against E-cadherin and then evaluated the expression of total and active $\beta$-catenin. Active $\beta$-catenin, which is not phosphorylated at Ser33/37 and Thr41, is functionally active in cell-to-cell adhesion and the canonical Wnt signaling pathway. A 48-h siRNA treatment revealed that a decrease in E-cadherin expression did not result in decreases in expression of total and active $\beta$-catenin (Fig. 6A). These findings suggest that Notch1 inhibits the expression of active and total $\beta$-catenin in an E-cadherin-independent manner. Then we questioned whether inhibition of expression of total and active $\beta$-catenin was a consequence of the activation of Notch signaling. The membrane-tethered Notch is transcriptionally inactive and considered biologically inert. To be biologically active, Notch1 requires intramembranous cleavage between amino acid Gly1743 and Val1744 mediated by the $\gamma$-secretase complex. We transfected A549 cells with biologically active Notch (pCS2-IVC-6mt) and membrane tethered Notch (pCS2- $\Delta \mathrm{EMV}-6 \mathrm{mt}$ ) and then evaluated the expression of total and active $\beta$-catenin. Expression of total and active $\beta$-catenin was inhibited by both Notch constructs (Fig. 6B).

Wnt/ $\beta$-catenin signaling is deregulated by the Notchl pathway. Since Notch1 is a transmembrane protein that relays signals from cellular interactions and interacts with $\beta$-catenin, one of the important components of adherens junctions, we reasoned that Notch1 is involved in the Wnt/ $\beta$-catenin signaling pathway. Active $\beta$-catenin, cyclin D1 and Axin2 are the representative markers of activation of $\mathrm{Wnt} / \beta$-catenin signaling. Markers for activation of Wnt/ $\beta$-catenin signaling were decreased by transduction with N1ICD (Fig. 6C). Since the $\mathrm{Wnt} / \beta$-catenin pathway is one of the important regulators of cell cycle progression, we evaluated whether N1ICD influences cell cycle propagation through regulation of the Wnt/ $\beta$-catenin pathway. Cell cycle progression of parental, control, and N1ICD-transduced cells was measured by flow cytometry after PI staining and comparisons were made to the proportion of cells in the $\mathrm{G} 2 / \mathrm{M}$ and $\mathrm{S}$ phases between the control and N1ICD-transduced cells at the same time points. There were no statistical differences in the cell cycle progression between the control and N1ICD-transduced A549 (Fig. 6D) and H1650 cells (data not shown).

\section{Discussion}

The adherens junction complex, in addition to its adhesive function of maintaining the integrity of epithelial cell-to-cell contacts, plays a crucial role in regulating Wnt/ $\beta$-catenin signaling. Loss of cadherin-mediated cell adhesion promotes Wnt $/ \beta$-catenin signaling, and deregulated $\mathrm{Wnt} / \beta$-catenin signaling due to unstable $\mathrm{E}$-cadherin $/ \beta$-catenin interaction induces expression of oncogenes such as $c-m y c$ and cyclin Dl $(22,23)$. Kwon et al $(15)$ showed that NTMIC interacts with $\beta$-catenin through an RAM domain and negatively regulates $\mathrm{Wnt} / \beta$-catenin signaling in a Numb-dependent manner, further complicating the signaling cascades mediated by adherens junctions.

The addition of any soluble ligands into culture media does not activate Notch1 signaling. To active Notch1 signaling, Notch1 ligands must be immobilized on a culture plate, aggregated with macromolecules and overexpressed on co-cultured ligand cells (24). Otherwise active Notch1 molecules have to be either transfected or transduced or culture medium has to be briefly depleted of calcium to dissociate Notch1 
A

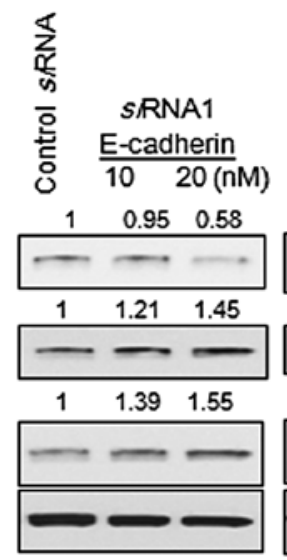

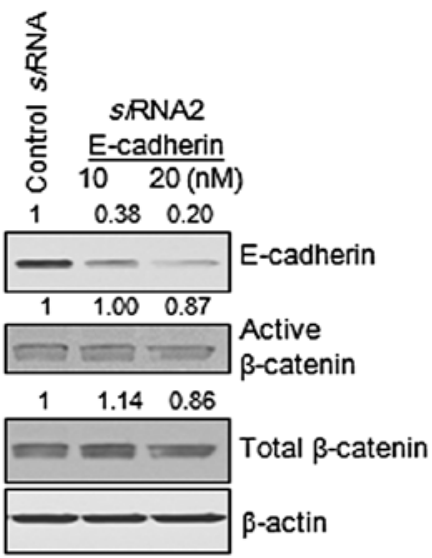

B

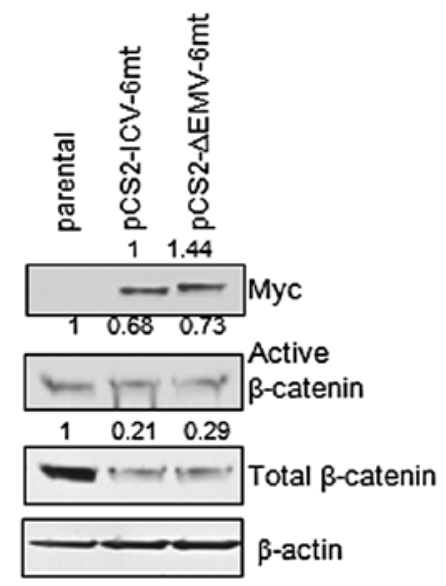

C

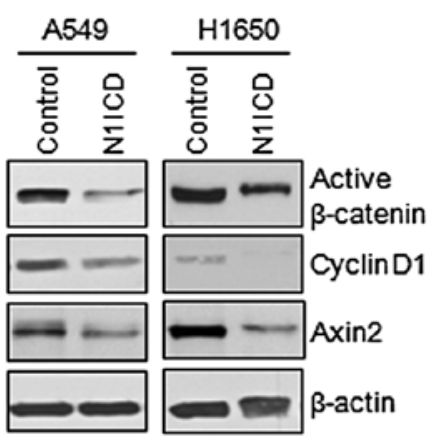

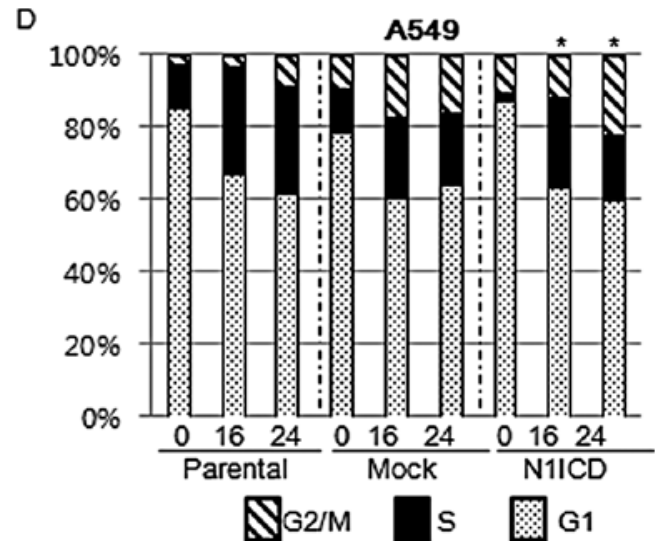

Figure 6. Downregulated expression of $\beta$-catenin by Notch1 intracellular domain (N1ICD) is E-cadherin independent. (A) A549 cells were treated by 2 independent siRNAs against E-cadherin, and expression of active and total $\beta$-catenin was evaluated by immunoblotting. Knockdown of E-cadherin did not decrease expression of total and active $\beta$-catenin. (B) Active Notch1 (pCS2-ICV-6mt) and membrane-tethered, biologically inert, Notch1 (pCS2- $\Delta$ EMV-6mt) were transfected into A549 cells followed by immunoblotting for active and total $\beta$-catenin. Decreased expression of active and total $\beta$-catenin was independent to Notch1 activity. (C) A549 and H1650 cells transduced with N1ICD or control vector were immunoblotted for the markers for Wnt/ $\beta$-catenin signaling, active $\beta$-catenin, Axin 2 and cyclin D1. Wnt/ $\beta$-catenin signaling was inhibited by N1ICD transduction. (D) Cell cycle progression of A549 parental, control, and N1ICD-transduced cells was measured by flow cytometry after PI staining. Cells were synchronized by $36 \mathrm{~h}$ of serum starvation. There were no statistical differences in the cell cycle progression between control and N1ICD transduced cells ( $\mathrm{P}>0.05)$. Comparisons were made to the proportion of cells in the G2/M and S phase between control and N1ICD transduced cells at the same time points. P-value was obtained by Student's t-test.

heterodimers (10). In the present study, we used the calcium chelating agent EDTA to induce N1ICD. Although multiple targets may be affected by calcium chelation, it is clear that calcium chelation induces the biologically active Notch1 molecule N1ICD, and propagates Notch1 signaling through transcriptional activation of downstream targets. During the period when N1ICD is detected by immunoblotting, we did not observe changes in the mRNA levels of E-cadherin, indicating that N1ICD may not directly repress E-cadherin expression (data not shown).

E-cadherin expression can be caused by promoter hypermethylation (25), CDH1 gene mutations (26), or induction of E-cadherin transcriptional repressors, including the bHLH family members Twist1 and Twist2, the E2A gene product E12/47, the Snail gene family of transcription factors, and the ZFH family repressors ZEB1 and ZEB2 (27). There are a few reports of the involvement of Notch1 signaling in the loss of E-cadherin and the EMT through upregulation of either Snail (SNAI1) $(28,29)$ or Slug (SNAI2) $(30)$. It has also been reported that Notch1 induces an EMT phenotype through activation of TGF- $\beta / \mathrm{Smad} 3$ pathways (31) or directly induces the expression of smooth muscle $\alpha$-actin (SMA) (32). Although there are two CSL consensus-binding sites in the SMA gene promoters $(32,33)$, we did not observe increased mRNA expression of SMA in N1ICD-transduced and EDTAexposed NSCLC cells (data not shown). We were not able to detect morphological differences among N1ICD-, control vector-transduced, and parental cells under a phase contrast microscope, suggesting that Notch1-mediated E-cadherin repression may be an early event in Notch1-mediated EMT.

There is mutual compensation between Notch1 and Wnt/ $\beta$-catenin signaling. c-Myc is a common target molecule of the Notch1 and Wnt/ $\beta$-catenin signaling pathways. Among the cyclin D family members, cyclin D1 is a target of the Wnt $/ \beta$-catenin pathway and cyclin D3 is a target of the Notch1 pathway. Notch1 has conflicting roles in $\mathrm{Wnt} / \beta$-catenin signaling. The physical interaction between Notch1 and $\beta$-catenin negatively regulates $\mathrm{Wnt} / \beta$-catenin signaling. In contrast, Notch1 participates in the deregulation of $\mathrm{Wnt} / \beta$-catenin signaling by inhibiting the expression of E-cadherin. Furthermore, N1ICD 
transduction was found to result in cell cycle progression and aneuploidy, which suggests another role for Notch1 in cancer progression. Sarmento et al (34) reported that forced induction of the N1ICD upregulated the expression of the $S$ phase kinaseassociated protein 2 (SKP2) and caused premature entry into $S$ phase. In the present study, transduction of cells with N1ICD decreased active $\beta$-catenin, cyclin D1 and Axin 2 expression, indicating that the active form of Notch1 acts as an inhibitor of the $\mathrm{Wnt} / \beta$-catenin pathway. However, negative regulation of Wnt/ $\beta$-catenin signaling through Notch1 did not result in a difference in cell cycle progression between the control vector and N1ICD-transduced cells. Taken together, activation of $\mathrm{Wnt} / \beta$-catenin signaling mediated by E-cadherin loss is compensated for by the inhibitory effect of Notch1 on $\beta$-catenin.

In conclusion, activation of Notch1 signaling destabilizes adherens junction by inhibition of E-cadherin and $\beta$-catenin expression. Transduction of the transcriptionally active domain of Notch1, namely N1ICD, into NSCLC cells decreased expression of E-cadherin through induction of a Snail family of E-cadherin repression complex. N1ICD also decreased the expression of $\beta$-catenin in an E-cadherin-independent manner, which resulted in a decrease in the expression of markers of activation of $\mathrm{Wnt} / \beta$-catenin. Despite the inhibition of Wnt/ $\beta$-catenin signaling in N1ICD-transduced cells, cells transduced with N1ICD showed no difference in cell cycle progression when compared with the control vector-transduced cells, suggesting that Notch1 signaling may compensate for the inhibition of the $\mathrm{Wnt} / \beta$-catenin pathway.

\section{Acknowledgements}

We thank Dr R. Kopan (Washington University) and Dr G. Jung (Seoul National University) for providing the Notch deletion/ tethered Notch construct and the MSCV-N1ICD construct, respectively. The present study was supported by an institutional grant from Yonsei University College of Medicine (6-2012-0104) awarded to Y.S.C.

\section{References}

1. Jemal A, Bray F, Center MM, Ferlay J, Ward E and Forman D: Global cancer statistics. CA Cancer J Clin 61: 69-90, 2011.

2. Kopan R and Ilagan MX: The canonical Notch signaling pathway: unfolding the activation mechanism. Cell 137: 216-233, 2009.

3. Gustafsson MV, Zheng X, Pereira T, et al: Hypoxia requires notch signaling to maintain the undifferentiated cell state. Dev Cell 9: 617-628, 2005.

4. Chen Y, De Marco MA, Graziani I, et al: Oxygen concentration determines the biological effects of NOTCH-1 signaling in adenocarcinoma of the lung. Cancer Res 67: 7954-7959, 2007.

5. Lim SO, Kim HS, Quan X, et al: Notch1 binds and induces degradation of Snail in hepatocellular carcinoma. BMC Biol 9: 83, 2011.

6. Joshi I, Minter LM, Telfer J, et al: Notch signaling mediates G1/S cell-cycle progression in T cells via cyclin D3 and its dependent kinases. Blood 113: 1689-1698, 2009.

7. Morimura T, Goitsuka R, Zhang Y, Saito I, Reth M and Kitamura D: Cell cycle arrest and apoptosis induced by Notch1 in B cells. J Biol Chem 275: 36523-36531, 2000.

8. Sriuranpong V, Borges MW, Ravi RK, et al: Notch signaling induces cell cycle arrest in small cell lung cancer cells. Cancer Res 61: 3200-3205, 2001.

9. Qi R, An H, Yu Y, et al: Notch1 signaling inhibits growth of human hepatocellular carcinoma through induction of cell cycle arrest and apoptosis. Cancer Res 63: 8323-8329, 2003.
10. Rand MD, Grimm LM, Artavanis-Tsakonas S, et al: Calcium depletion dissociates and activates heterodimeric notch receptors. Mol Cell Biol 20: 1825-1835, 2000.

11. King AM, Van der Put E, Blomberg B and Riley RL: Accelerated Notch-dependent degradation of E47 proteins in aged B cell precursors is associated with increased ERK MAPK activation. J Immunol 178: 3521-3529, 2007.

12. Bryant DM and Mostov KE: From cells to organs: building polarized tissue. Nat Rev Mol Cell Biol 9: 887-901, 2008.

13. Behrens J, Vakaet L, Friis R, et al: Loss of epithelial differentiation and gain of invasiveness correlates with tyrosine phosphorylation of the E-cadherin/beta-catenin complex in cells transformed with a temperature-sensitive v-SRC gene. J Cell Biol 120: 757-766, 1993.

14. Taddei ML, Chiarugi $\mathrm{P}$, Cirri $\mathrm{P}$, et al: Beta-catenin interacts with low-molecular-weight protein tyrosine phosphatase leading to cadherin-mediated cell-cell adhesion increase. Cancer Res 62: 6489-6499, 2002.

15. Kwon C, Cheng P, King IN, et al: Notch post-translationally regulates $\beta$-catenin protein in stem and progenitor cells. Nat Cell Biol 13: 1244-1251, 2011.

16. Schroeter EH, Kisslinger JA and Kopan R: Notch-1 signalling requires ligand-induced proteolytic release of intracellular domain. Nature 393: 382-386, 1998.

17. DuPage M, Dooley AL and Jacks T: Conditional mouse lung cancer models using adenoviral or lentiviral delivery of Cre recombinase. Nat Protoc 4: 1064-1072, 2009.

18. van Tetering G, van Diest P, Verlaan I, van der Wall E, Kopan R and Vooijs M: Metalloprotease ADAM10 is required for Notch1 site 2 cleavage. J Biol Chem 284: 31018-31027, 2009.

19. Hazan RB, Qiao R, Keren R, Badano I and Suyama K: Cadherin switch in tumor progression. Ann NY Acad Sci 1014: 155-163, 2004.

20. Bremnes RM, Veve R, Gabrielson E, et al: High-throughput tissue microarray analysis used to evaluate biology and prognostic significance of the E-cadherin pathway in non-small-cell lung cancer. J Clin Oncol 20: 2417-2428, 2002.

21. Sahlgren C, Gustafsson MV, Jin S, Poellinger L and Lendahl U: Notch signaling mediates hypoxia-induced tumor cell migration and invasion. Proc Natl Acad Sci USA 105: 6392-6397, 2008.

22. He TC, Sparks AB, Rago C, et al: Identification of c-MYC as a target of the APC pathway. Science 281: 1509-1512, 1998.

23. Tetsu $\mathrm{O}$ and McCormick F: Beta-catenin regulates expression of cyclin D1 in colon carcinoma cells. Nature 398: 422-426, 1999.

24. Moloney DJ, Panin VM, Johnston SH, et al: Fringe is a glycosyltransferase that modifies Notch. Nature 406: 369-375, 2000.

25. Graff JR, Herman JG, Lapidus RG, et al: E-cadherin expression is silenced by DNA hypermethylation in human breast and prostate carcinomas. Cancer Res 55: 5195-5199, 1995.

26. Berx G, Becker KF, Höfler H and van Roy F: Mutations of the human E-cadherin (CDH1) gene. Hum Mutat 12: 226-237, 1998.

27. Schmalhofer O, Brabletz S and Brabletz T: E-cadherin, betacatenin, and ZEB1 in malignant progression of cancer. Cancer Metastasis Rev 28: 151-166, 2009.

28. Saad S, Stanners SR, Yong R, Tang O and Pollock CA: Notch mediated epithelial to mesenchymal transformation is associated with increased expression of the Snail transcription factor. Int J Biochem Cell Biol 42: 1115-1122, 2010.

29. Matsuno Y, Coelho AL, Jarai G, Westwick J and Hogaboam CM: Notch signaling mediates TGF- $\beta 1$-induced epithelial-mesenchymal transition through the induction of Snail. Int J Biochem Cell Biol 44: 776-789, 2012.

30. Niessen K, Fu Y, Chang L, Hoodless PA, McFadden D and Karsan A: Slug is a direct Notch target required for initiation of cardiac cushion cellularization. J Cell Biol 182: 315-325, 2008.

31. Aoyagi-Ikeda K, Maeno T, Matsui $\mathrm{H}$, et al: Notch induces myofibroblast differentiation of alveolar epithelial cells via transforming growth factor-\{beta\}-Smad3 pathway. Am J Respir Cell Mol Biol 45: 136-144, 2011.

32. Noseda M, Fu Y, Niessen K, et al: Smooth muscle alpha-actin is a direct target of Notch/CSL. Circ Res 98: 1468-1470, 2006.

33. Tang Y, Urs S, Boucher J, et al: Notch and transforming growth factor-beta (TGFbeta) signaling pathways cooperatively regulate vascular smooth muscle cell differentiation. J Biol Chem 285: 17556-17563, 2010.

34. Sarmento LM, Huang H, Limon A, et al: Notch1 modulates timing of G1-S progression by inducing SKP2 transcription and p27 Kip1 degradation. J Exp Med 202: 157-168, 2005. 